\title{
Representación finita de variedades compactas
}

\author{
Carlos Mario Parra*, Johany Suárez Ramírez \\ Universidad Nacional de Colombia, Escuela de Matemáticas, Medellín, Colombia.
}

Resumen. Un logro notable de la topología algorítmica es el resultado de A.A. Márkov sobre la insolubilidad del problema del homeomorfismo para variedades. Posteriormente, Boone, Haken y Poénaru extendieron la idea original de Márkov al caso de variedades suaves cerradas. Una primera dificultad era la introducción de una representación finita de una variedad diferenciable o combinatórica que la describiese de forma natural. En este trabajo extendemos dicha representación a variedades suaves compactas y proponemos una definición de variedad suave representable.

Palabras clave: Computabilidad y teoría de la recursión, topología algorítmica, variedades suaves.

MSC2010: 03D80, 57N13, 57N15, 57Q15, 57R55.

\section{Finite representation of compact manifolds}

Abstract. A remarkable achievement of algorithmic topology is A.A. Markov's theorem on the unsolvability of the homeomorphism problem for manifolds. Boone, Haken and Poénaru extended Markov's original proof to the case of closed smooth manifolds. One of their initial difficulties was the introduction of a natural finite representation of a differentiable and/or combinatorial manifold. In this paper we extend this representation to compact smooth manifolds and propose an extension to smooth manifolds.

Keywords: Computability and recursion theory, algorithmic topology, smooth manifolds.

\footnotetext{
*E-mail: cmparra@unal.edu.co

Recibido: 26 de marzo de 2015, Aceptado: 18 de agosto de 2015.

Para citar este artículo: C.M Parra, J. Suárez Ramírez, Representación finita de variedades compactas, Rev. Integr. Temas Mat. 33 (2015), No. 2, 97-105.
} 


\section{Introducción}

Una de las preguntas básicas en topología es el siguiente problema de decisión:

\section{PROBLEMA DEL HOMEOMORFISMO}

Entrada: triangulaciones $\mathcal{K}$ y $\mathcal{L}$ cuyos poliedros $M=|\mathcal{K}|$ y $N=|\mathcal{L}|$ son $n$-variedades. Pregunta: ¿ $M$ y $N$ son homeomorfos?

Una restricción natural del problema anterior consiste en fijar uno de los poliedros, digamos $M=|\mathcal{K}|$, y buscar un algoritmo que, dada cualquier triangulación $\mathcal{L}$, determine si $N=|\mathcal{L}|$ es homeomorfo a $M$. El teorema clásico de clasificación de superficies compactas ofrece una respuesta positiva a los anteriores problemas para el caso $n=2$. En la actualidad se ha establecido que el problema del homeomorfismo es soluble para 3-variedades cerradas orientables irreducibles [2]. Sin embargo, el caso general para 3 -variedades cerradas orientables permanece abierto [1], aunque avances recientes apoyan una visión optimista de una solución general. En dimensiones superiores, A.A. Márkov [8] mostró el siguiente

Teorema 1.1 (Márkov, 1958). Para $n \geq 4$ es posible construir una $n$-variedad $M$ tal que el problema de reconocer dicha variedad es algorítmicamente insoluble.

Como consecuencia de lo anterior, obtenemos:

Corolario 1.2. El problema del homeomorfismo de $n$-variedades es insoluble para $n \geq 4$.

Continuando en esta dirección, W.W. Boone, W. Haken y V. Poénaru [3] extendieron el trabajo de Márkov a variedades suaves.

Teorema 1.3. Para todo $n \geq 4$ existe una clase recursiva $\mathcal{C}_{n}(D)$ de representaciones finitas de $n$-variedades, dotadas con una estructura suave y una correspondiente estructura combinatórica compatible, tal que el problema del difeomorfismo asociado a $\mathcal{C}_{n}(D)$ es insoluble.

Una dificultad inherente a la discusión anterior es cómo representar la estructura diferenciable de una variedad. Con base en métodos desarrollados por J. Nash [13], Boone et al. propusieron una noción de representación algebraica para variedades cerradas. Esto a su vez abrió el camino a resultados de insolubilidad en la categoría de variedades suaves. Una ilustración notable es el siguiente resultado de S. P. Nóvikov, ver [7, p. 329-332].

Teorema 1.4 (Nóvikov, 1974). Para $n \geq 5$ es imposible reconocer cualquier $n$-variedad en la categoría de variedades suaves. En particular, es imposible reconocer $\mathbb{S}^{n}$.

Una variación del teorema anterior se debe a A. Nabutovsky [11], quien probó que es imposible reconocer $\mathbb{S}^{n}, n \geq 5$, en la clase de hipersuperficies algebraicas no-singulares en $\mathbb{R}^{n+1}$.

Teorema 1.5. Para todo $n \geq 5$ no existe un algoritmo que resuelva el siguiente problema: dado un vector de coeficientes de un polinomio racional $p: \mathbb{R}^{n+1} \rightarrow \mathbb{R}$ tal que el conjunto cero $Z(p)$ de $p$ es no vacío, compacto y no-singular, ¿es $Z(p)$ difeomorfo a $\mathbb{S}^{n}$ ?

[Revista Integración 
Por otro lado, en $1954 \mathrm{~W}$. Haken propuso un algoritmo que permite determinar la trivialidad de un nudo en $\mathbb{R}^{3}$. En contraste, A. Nabutovsky y S. Weinberger [12] mostraron que el problema de decidir si se puede desanudar un nudo $n$-dimensional es insoluble para $n \geq 3$.

Teorema 1.6. Para cualquier $n \geq 3$ fijo, no existe un algoritmo que decida la trivialidad de un $n$-nudo $k: \mathbb{S}^{n} \rightarrow \mathbb{R}^{n+2}$ ( $k$ puede ser un embebimiento PL o suave de $\mathbb{S}^{n}$ en $\mathbb{R}^{n+2}$ ).

Para un estudio más detallado de problemas indecidibles en topología y geometría diferencial, el lector interesado puede consultar Soare [14].

Los anteriores resultados sugieren la pregunta natural de cómo capturar la estructura diferenciable de una $n$-variedad suave con frontera. O, más generalmente, cómo representar variedades suaves no compactas. Un primer intento sería generalizar la demostración original de Boone et al. a variedades compactas. Sin embargo, los resultados de Nash involucrados en dicha prueba, específicamente los lemas 1 y 2 [13, p. 408-409], se enunciaron originalmente para variedades cerradas y no luce claro cómo generalizarlos a otro tipo de variedades. Consideramos que un método alternativo consiste en usar el concepto de doble de una variedad y aplicar un teorema clásico de extensión de J.H.C. Whitehead.

\section{Triangulaciones suaves}

A continuación desarrollamos la notación y las nociones estándar de topología PL y topología suave requeridas. Referimos al lector a Bryant [4] y Munkres [10] para más detalles.

Empezamos por recordar que un poliedro $K$ es la unión de los simplejos $\sigma$ de un complejo simplicial $\mathcal{K}$, con la notación usual $K=|\mathcal{K}|$. De otro lado, dados complejos $\mathcal{K}$ y $\mathcal{K}^{\prime}$, decimos que $\mathcal{K}^{\prime}$ es una subdivisión de $\mathcal{K}$ si $\left|\mathcal{K}^{\prime}\right|=|\mathcal{K}|$ y cada simplejo de $\mathcal{K}^{\prime}$ está contenido en alguno de $\mathcal{K}$. Dado un vértice $v \in|\mathcal{K}|$, definimos la estrella como

$$
\operatorname{st}(v, \mathcal{K})=\bigcup_{v \in \sigma} \sigma
$$

Lema 2.1. Si $\mathcal{K}_{1}$ y $\mathcal{K}_{2}$ son complejos tales que $\left|\mathcal{K}_{1}\right|=\left|\mathcal{K}_{2}\right|$, entonces $\mathcal{K}_{1}$ y $\mathcal{K}_{2}$ tienen una subdivisión simplicial común.

Lema 2.2. Si $\mathcal{K}_{1}$ y $\mathcal{K}_{2}$ son complejos finitos, entonces existen subdivisiones $\mathcal{K}_{1}^{\prime}$ y $\mathcal{K}_{2}^{\prime}$ de $\mathcal{K}_{1}$ y $\mathcal{K}_{2}$, respectivamente, tales que $\mathcal{K}_{1} \cup \mathcal{K}_{2}$ es un complejo simplicial.

Definición 2.3. Sean $\mathcal{K}$ y $\mathcal{L}$ complejos simpliciales y $f:|\mathcal{K}| \rightarrow|\mathcal{L}|$ una función simplicial (i.e., $f$ envía simplejos de $\mathcal{K}$ en simplejos de $\mathcal{L}$ ). Decimos que $f$ es:

(a) lineal, si $f(x)=f\left(\sum \alpha_{i} v_{i}\right)=\sum \alpha_{i} f\left(v_{i}\right)$, donde $\alpha_{i} \geq 0$ y $\sum \alpha_{i}=1$;

(b) lineal a tramos (PL), si existe una subdivisión $\mathcal{K}^{\prime}$ de $\mathcal{K}$ tal que $f:\left|\mathcal{K}^{\prime}\right| \rightarrow|\mathcal{L}|$ es lineal;

(c) un isomorfismo lineal, si $f$ es un homeomorfismo y es lineal. 
Definición 2.4. Un poliedro $M \subseteq \mathbb{R}^{m}$ es una $n$-variedad PL si para cada $p \in M$ existe una $p$-vecindad cerrada PL-homeomorfa a un $n$-simplejo.

Suponemos que el lector tiene familiaridad con la noción de variedad suave $(M, \mathcal{U})$. Específicamente, recordamos que la notación anterior significa que $M$ es una variedad topológica y $\mathcal{U}$ es un atlas maximal de clase $C^{\infty}$. Similarmente, si $(M, \mathcal{U})$ y $(N, \mathcal{V})$ son variedades suaves, una función $f: M \rightarrow N$ es suave (de clase $C^{r}$ ) si dadas cartas $h$ y $g$ en $\mathcal{U}$ y $\mathcal{V}$, respectivamente, la función $g \circ f \circ h^{-1}$ es un difeomorfismo adecuado.

Definición 2.5. (a) Una triangulación de $X$ es un homeomorfismo $t:|\mathcal{K}| \rightarrow X$.

(b) Dos triangulaciones $t$ y $t^{\prime}$ son equivalentes si existe un PL-homeomorfismo $h:\left|\mathcal{K}^{\prime}\right| \rightarrow|\mathcal{K}|$ tal que $t^{\prime} \circ h=t$.

(c) Si $X=|\mathcal{K}|$ es una $n$-variedad $\mathrm{PL},(X,|\mathcal{K}|, t)$ es una variedad combinatórica y $t$ es una estructura combinatórica (estructura PL) para $X$.

Definición 2.6 (Cairns, [5] y [6]). Una estructura combinatórica $t:|\mathcal{K}| \rightarrow M$ es compatible con $\mathcal{U}$ si para todo $m$-simplejo $\sigma \in \mathcal{K}$ existe una carta $\varphi: W \rightarrow \mathbb{H}^{n}$ en $\mathcal{U}$ tal que

$$
t(\sigma) \subseteq W \quad \text { y } \quad \varphi(t(\sigma)) \text { es un } m \text {-simplejo en } \mathbb{H}^{n},
$$

donde $\mathbb{H}^{n}$ denota el hiperplano superior $n$-dimensional

$$
\mathbb{H}^{n}=\left\{\mathbf{x} \in \mathbb{R}^{n} \mid x_{k} \geq 0\right\} .
$$

Cuando $t$ es compatible con $\mathcal{U}$, se dice que $t$ es una triangulación suave de $M$.

Una noción equivalente de triangulación suave aparece en Whitehead [15]. Para una versión más sencilla recomendamos el apéndice II de Milnor [9]. Para la equivalencia de estas nociones ver el Teorema 8.4 en Munkres [10]. El siguiente resultado es un teorema clásico debido a S.S. Cairns y J.H.C. Whitehead.

Teorema 2.7. Toda variedad suave admite una estructura combinatórica compatible.

Una extensión de una triangulación suave $f: J \rightarrow \partial M$ es una triangulación suave $g: L \rightarrow M$ tal que $g^{-1} \circ f$ es un isomorfismo lineal sobre un subcomplejo de $L$.

Teorema 2.8. Sea $(M, \mathcal{U})$ una variedad suave. Cualquier triangulación suave de $\partial M$ se puede extender a una triangulación suave de $M$.

También haremos uso del siguiente resultado (ver Munkres [10, Theorem 10.5]).

Proposición 2.9. Sea $M$ una variedad suave. Si $f:|\mathcal{K}| \rightarrow M$ y $g:|\mathcal{L}| \rightarrow M$ son triangulaciones suaves de $M$, entonces existen subdivisiones de $\mathcal{K}$ y $\mathcal{L}$ que son linealmente isomorfas. De hecho, existen triangulaciones suaves $f^{\prime}:\left|\mathcal{K}^{\prime}\right| \rightarrow M$ y $g^{\prime}:\left|\mathcal{L}^{\prime}\right| \rightarrow M$ tales que $\left(f^{\prime}\right)^{-1} \circ g^{\prime}$ es un isomorfismo lineal de $\mathcal{L}^{\prime}$ con $\mathcal{K}^{\prime}$.

[Revista Integración 


\section{Representación finita de estructuras suaves}

Para extender los resultados de insolubilidad a variedades suaves, surge la dificultad de cómo representar, mediante una notación finita, la estructura suave correspondiente. Un primer intento podría ser mediante una triangulación. Pero, en general, no existe un algoritmo que permita reconocer si un complejo simplicial representa una $n$-variedad, para $n \geq 5$. Una suerte similar corren los enfoques naturales análogos (matrices de incidencia, simplejos abstractos, etc.). De modo que se requiere algo más elaborado. Un enfoque exitoso en este sentido es la propuesta de Boone et al. (ver sección 3.2 en [3]). En dicho tratamiento, los autores imponen las siguientes condiciones mínimas necesarias sobre una representación finita $\mathfrak{M}$ de una variedad suave:

(a) $\mathfrak{M}$ es una notación finita, i.e., es una cadena finita en algún lenguaje.

(b) Existe un algoritmo para determinar si una cadena en este lenguaje es una representación finita.

(c) A cada presentación finita $\mathfrak{M}$ le corresponde una única variedad $M(\mathfrak{M})$ representada por $\mathfrak{M}$.

(d) $\mathfrak{M}$ describe la variedad $M(\mathfrak{M})$ en una forma natural. Esto es, cualquier representación finita $\mathfrak{M}$ debe generar una triangulación $\Delta$ y un atlas suave compatible $\mathcal{U}$ para la variedad $M(\mathfrak{M})$.

Boone et al. definen una representación algebraica finita $\mathfrak{M}$ de una n-variedad cerrada con una estructura suave y una correspondiente estructura combinatórica compatible como la colección ordenada

$$
\mathfrak{M}=\left(x_{1}, \ldots, x_{q} ; \quad \mathbf{v}_{1}, \ldots, \mathbf{v}_{s} ; \theta ; \quad \mathbf{i}_{1}, \ldots, \mathbf{i}_{s} ; \quad h_{1}, \ldots, h_{s}\right),
$$

con las siguientes propiedades:

(I) $x_{1}, \ldots, x_{q}$ es un conjunto de letras, llamadas variables coordenadas, o simplemente, coordenadas. $\mathbb{E}^{q}(\mathfrak{M})$ denota el $q$-espacio euclidiano representado por $\mathfrak{M}$, el espacio euclidiano de dimensión $q$ con coordenadas $x_{1}, \ldots, x_{q}$.

(II) $\mathbf{v}_{1}, \ldots, \mathbf{v}_{s}$ son vectores en $\mathbb{E}^{q}(\mathfrak{M})$ con componentes racionales, distintos por pares; $\theta$ es un complejo abstracto finito $n$-dimensional con vértices $\mathbf{v}_{1}, \ldots, \mathbf{v}_{s}$. Se requiere las siguientes propiedades:

(IIa) Si $\left\{\mathbf{v}_{j_{0}}, \ldots, \mathbf{v}_{j_{m}}\right\} \in \theta$, entonces $\mathbf{v}_{j_{1}}, \ldots, \mathbf{v}_{j_{m}}$ están en posición general, i.e., en $\mathbb{E}^{q}(\mathfrak{M})$ existe un $m$-simplejo $\sigma_{m}$ con vértices $\mathbf{v}_{j_{0}}, \ldots, \mathbf{v}_{j_{m}}$.

(IIb) El conjunto de todos aquellos simplejos que corresponde a elementos de $\theta$ en el sentido de (IIa) es un complejo simplicial rectilíneo en $\mathbb{E}^{q}(\mathfrak{M})$, el complejo simplicial representado por $\mathfrak{M}$, denotado por $\Delta(\mathfrak{M})$. Además, $\Delta(\mathfrak{M})$ tiene frontera vacía.

Vol. 33, No. 2, 2015] 
(III) $\mathbf{i}_{k}(k=1, \ldots, s)$ es una $n$-tupla de enteros positivos $i_{1 k}<i_{2 k}<\cdots<i_{n k} \leq q$ tal que la estrella (compacta) $s t\left(\mathbf{v}_{k}, \Delta(\mathfrak{M})\right)$ proyecta inyectivamente en el espacio coordenado $\mathbb{E}^{n}\left(\mathbf{i}_{k}\right)$ con coordenadas $x_{i_{1 k}}, \ldots, x_{i_{n k}}$. Es decir, el mapeo $\iota_{k}: \mathbb{E}^{q}(\mathfrak{M}) \rightarrow \mathbb{E}^{n}\left(\mathbf{i}_{k}\right)$, dado por $\iota_{k}\left(a_{1}, \ldots, a_{q}\right)=\left(a_{i_{1 k}}, \ldots, a_{i_{n k}}\right)$, induce un PL-homeomorfismo de $\operatorname{st}\left(\mathbf{v}_{k}, \Delta(\mathfrak{M})\right)$ en $\mathbb{E}^{n}\left(\mathbf{i}_{k}\right)$. Lo anterior hace evidente que $\Delta(\mathfrak{M})$ es una $n$-variedad combinatórica.

(IV) Para $k=1, \ldots, s, h_{k}$ es un homeomorfismo del interior de $\operatorname{st}\left(\mathbf{v}_{k}, \Delta(\mathfrak{M})\right)$ en el $n$-subespacio $\mathbb{E}^{n}$ de $\mathbb{E}^{q}(\mathfrak{M})$ con coordenadas $x_{1}, \ldots, x_{n}$. Estos homeomorfismos forman un atlas suave $\mathcal{U}(\mathfrak{M})$ en $|\Delta(\mathfrak{M})|$, el atlas representado por $\mathfrak{M}$, y se describen por un conjunto finito de ecuaciones algebraicas.

Las ecuaciones algebraicas están codificadas por una matriz simétrica $\mathbf{L}$ de orden $q \times q$ y un vector $\mathbf{u}$ de orden $1 \times q$, cuyas entradas son polinomios en las variables $x_{1}, \ldots, x_{q}$ con coeficientes racionales. Aquí Boone et al. aplican las técnicas desarrolladas por Nash [13]. Los homeomorfismos se pueden interpretar como el mapeo que envía cada punto del poliedro $|\Delta|$ al punto más cercano de una hoja de aproximación $\mathcal{B}$ de una variedad algebraica y luego proyecta dicho punto en un $n$-subespacio de $\mathbb{E}^{q}(\mathfrak{M})$. De otro lado, $\mathbf{u}$ aproxima el vector distancia de cualquier punto en una vecindad de $\mathcal{B}$ al punto más cercano en $\mathcal{B}$; la matriz $\mathbf{L}$ aproxima la matriz de componentes del tensor que proyecta cada vector en el plano $(q-n)$-dimensional normal a $\mathcal{B}$. Para más detalles técnicos de esta construcción, ver [3, p. 58-59].

La $n$-variedad suave combinatórica $M(\mathfrak{M})$, representada por $\mathfrak{M}$, es el poliedro $|\Delta(\mathfrak{M})|$ junto con la estructura combinatórica $\Delta(\mathfrak{M})$ y la estructura suave generada por el atlas $\mathcal{U}(\mathfrak{M})$.

El resultado principal de Boone et al. [3, Theorem 4] es el siguiente teorema fundamental.

Teorema 3.1. (a) Para toda n-variedad suave cerrada $M$ existe una representación algebraica finita $\mathfrak{M}$ tal que $M(\mathfrak{M})$ es difeomorfa a $M$.

(b) El concepto de representación algebraica satisface (a), (b) y (c). Además, en cuanto a (d), se cumple que dada la representación $\mathfrak{M}$ el atlas suave $\mathcal{U}(\mathfrak{M})$ es computable. Esto es, existe un algoritmo que, dados un racional positivo $\epsilon$ y un punto racional $\mathbf{p} \in \operatorname{st}\left(\mathbf{v}_{k}, \Delta(\mathfrak{M})\right)$, determina las componentes de un punto racional $h_{k}^{\#}(\mathbf{p}) \in \mathbb{R}^{n}$ tal que

$$
\left|h_{k}^{\#}(\mathbf{p})-h(\mathbf{p})\right|<\epsilon .
$$

\section{Representación finita de variedades con frontera}

Una pregunta natural que surge de los resultados anteriores es cómo extender el método de representación a variedades compactas (posiblemente con frontera). Nuestra idea es usar el concepto del doble de una variedad, el cual introducimos a continuación. Sean $M$ y $N$ variedades suaves con frontera y $h: \partial M \rightarrow \partial N$ un difeomorfismo. Denotamos por $M \amalg N=M \cup_{h} N$ a la variedad que resulta al identificar $x \sim h(x)$, para cada $x \in \partial M$. Se puede ver entonces que $M \amalg N$ es una variedad suave cerrada que contiene copias de $M$ y $N$. Específicamente, las inyecciones naturales $i: M \rightarrow M \amalg N$ y $j: N \rightarrow M \amalg N$ son embebimientos. En el caso particular en que $M=N$ y $h$ es la identidad, obtenemos la variedad $D(M)=M \cup_{\text {id }} M$ denominada el doble de $M$. 


\subsection{Definición de la representación}

Para extender la propuesta de Boone et al. a variedades suaves compactas con frontera, definimos una representación (algebraica) finita como la tupla $\mathcal{R}=\langle\Delta, \mathfrak{M}\rangle$ que satisface las siguientes condiciones:

(i) $\mathfrak{M}$ es una representación finita de una variedad suave cerrada en el sentido anterior. Hacemos $\mathbb{E}^{q}(\mathcal{R})=\mathbb{E}^{q}(\mathfrak{M})$.

(ii) $\Delta$ es un complejo simplicial abstracto cuyos vértices $\mathbf{w}_{1}, \ldots, \mathbf{w}_{t}$ denotan vectores en $\mathbb{E}^{q}(\mathfrak{M})$ con coordenadas racionales. De un modo similar al numeral (II), exigimos que la realización de $\Delta$ en $\mathbb{E}^{q}(\mathcal{R})$ determine un complejo rectilíneo con frontera, que denotaremos por $\Delta(\mathcal{R})$. Finalmente, $\Delta(\mathcal{R})$ es un subcomplejo del complejo $\Delta(\mathfrak{M})$ determinado por la representación $\mathfrak{M}$.

(iii) Como en (III), para cada vértice $\mathbf{w}_{k}$ la estrella $\mathrm{st}\left(\mathbf{w}_{k}, \Delta(\mathcal{R})\right)$ se proyecta inyectivamente mediante un PL-homeomorfismo en un subespacio de $\mathbb{E}^{q}(\mathcal{R})$, lo cual muestra que el complejo $\Delta(\mathcal{R})$ es una variedad combinatórica con frontera.

(iv) Para cada vértice $\mathbf{w}_{k}$ restringimos las cartas del atlas $\mathcal{U}(\mathfrak{M})$ a las estrellas abiertas $\operatorname{st}\left(\mathbf{v}_{k}, \Delta(\mathcal{R})\right)$, con $k=1, \ldots, t$. Estas restricciones determinan un atlas suave sobre el poliedro $|\Delta(\mathcal{R})|$, el cual se denota por $\mathcal{U}(\mathcal{R})$.

Finalmente, la $n$-variedad suave y combinatórica $M(\mathcal{R})$ representada por $\mathcal{R}$ es el poliedro $|\Delta(\mathcal{R})|$ junto con la estructura combinatórica $\Delta(\mathcal{R})$ y el atlas suave $\mathcal{U}(\mathcal{R})$. Se verifica directamente que esta noción de representación satisface las condiciones (a)-(d) requeridas por Boone et al. Específicamente, en cuanto a la condición (d), el hecho de restringir el atlas computable $\mathcal{U}(\mathfrak{M})$ a las estrellas abiertas del poliedro $|\Delta(\mathcal{R})|$ provoca que el atlas generado también sea computable.

\subsection{Existencia de la representación}

Mostraremos la siguiente extensión del teorema fundamental de Boone et al.

Teorema 4.1. Para toda $n$-variedad suave compacta $M$ existe una representación algebraica finita $\mathcal{R}$ tal que la variedad $M(\mathcal{R})$ es difeomorfa a $M$. Además, $\mathcal{R}$ determina una estructura combinatórica para $M$ y una correspondiente estructura suave compatible. Por último, el atlas suave $\mathcal{U}(\mathcal{R})$ es computable en el sentido del teorema fundamental.

Demostración. Por el Teorema 3.1, existe una representación $\mathfrak{M}_{1}$ para el doble $D(M)$ de $M$, la cual determina un poliedro $P:=\left|\Delta\left(\mathfrak{M}_{1}\right)\right|$ cuyo atlas asociado es computable. Denotamos por $f:|\mathcal{K}| \rightarrow P$ la triangulación suave asociada a $\mathfrak{M}_{1}$.

Por otro lado, dado que $D(M)$ posee una copia difeomorfa de $M$, existe un embebimiento $j: M \rightarrow P$ tal que $Q:=j(M)$ es una subvariedad suave de $P$. Veamos que una modificación apropiada de $Q$ nos permite generar una representación algebraica para $M$.

Por el teorema de Cairns-Whitehead (Teorema 2.7), existe una triangulación suave de $Q$. Restringiendo esta triangulación a $\partial Q \cong \partial M$, y luego aplicando el Teorema 2.8, podemos extenderla a una triangulación suave $g:|\mathcal{L}| \rightarrow P$.

Vol. 33, No. 2, 2015] 
Aplicando la Proposición 2.9, existe una subdivisión común a las triangulaciones $f$ y $g$, que denotamos por $h:|\mathcal{J}| \rightarrow P$. Notemos que $\mathcal{J}$ es un subcomplejo simplicial de $\mathcal{K}$ y (también de $\mathcal{L}$ ).

Como en la demostración del teorema fundamental en [3], sin pérdida de generalidad podemos suponer que los vértices de $\mathcal{J}$ son vectores en $\mathbb{E}^{q}\left(\mathfrak{M}_{1}\right)$ con componentes racionales. Ahora reemplazamos $\mathcal{K}$ por $\mathcal{J}$ y restringimos el atlas $\mathcal{U}\left(\mathfrak{M}_{1}\right)$ a los simplejos de $\mathcal{J}$. De este modo, obtenemos otra representación algebraica $\mathfrak{M}$ para $D(M)$. Por último, como $Q$ es subpoliedro de $|\mathcal{L}|$, tomamos $\Delta$ como el subcomplejo simplicial de $\mathcal{J}$ asociado a $Q=j(M) \cong M$. Finalmente, hacemos $\mathcal{R}=\langle\Delta, \mathfrak{M}\rangle$.

Es natural buscar una extensión de representación algebraica finita para variedades no compactas. Proponemos la siguiente definición.

Definición 4.2. Sea $M$ una variedad suave. Decimos que $M$ es representable si existe una familia recursivamente enumerable $\left\{\mathcal{R}_{k}\right\}$ de representaciones tales que

$$
M=\bigcup_{k=1}^{\infty} M\left(\mathcal{R}_{k}\right)
$$

En otras palabras, $M$ es representable si $M$ es la unión de una familia contable $\left\{M_{k}\right\}$ de subvariedades compactas tal que existe un programa (máquina de Turing) $\mathcal{T}$ que genera sistemáticamente una representación algebraica para cada $M_{k}$. Podemos interpretar el programa $\mathcal{T}$ como una representación finita de $M$.

En el sentido de la definición anterior, es fácil ver que $\mathbb{R}^{n}, n \geq 1$, con su estructura suave usual, es una variedad representable. De otro lado, ya que bajo difeomorfismo existen infinitos tipos de variedades compactas conexas, un argumento de conteo muestra que existe una familia no contable de variedades suaves compactas. Por lo tanto, existe una familia no enumerable de variedades no representables.

\section{Referencias}

[1] Aschenbrenner M., Friedl S. and Wilton H., "Decision problems for 3-manifolds and their fundamental groups", Department of Mathematics, UCLA.

http://www.math.ucla.edu/ matthias/publications.html [citado 11 de agosto de 2015].

[2] Bessières L., Besson G., Maillot S., Boileau M. and Porti J., Geometrisation of 3-manifolds, EMS Tracts in Mathematics, 13, European Mathematical Society (EMS), Zürich, 2010.

[3] Boone W.W., Haken W. and Poénaru V., "On recursively unsolvable problems in topology and their classification", in Contributions to Math. Logic (Colloquium, Hannover, 1966) (ed. Schmidt H.A. et al.), North-Holland (1968), 37-74.

[4] Bryant J.L., "Piecewise linear topology", in Handbook of Geometric Topology (ed. Daverman R.J. and Sher R.B.), North-Holland (2002), 219-259.

[5] Cairns S.S., "Triangulation of the manifold of class one", Bull. Amer. Math. Soc. 41 (1935), No. 8, 549-552.

[Revista Integración 
[6] Cairns S.S., "A simple triangulation method for smooth manifolds", Bull. Amer. Math. Soc. 67 (1961), No. 4, 389-390.

[7] Chernavsky A.V. and Leksine V.P., "Unrecognizability of manifolds", Ann. Pure Appl. Logic. 141 (2006), No. 3, 325-335.

[8] Markov A.A., "Insolubility of the problem of homeomorphy" (Russian), in Proc. Internat. Congress Math. 1958 (ed. Todd J.A.), Cambridge Univ. Press, New York (1960), 300-306.

[9] Milnor J.W., "On the relationship between differentiable manifolds and combinatorial manifolds" (unpublished notes), Princeton University (1956). Department of Mathematics, Texas Christian University.

http://faculty.tcu.edu/gfriedman/notes/milnor3.pdf [citado 11 de agosto de 2015].

[10] Munkres J.R., Elementary Differential Topology, Princeton University Press, Princeton, NJ, 1966.

[11] Nabutovsky A., "Einstein structures: Existence versus uniqueness", Geom. Funct. Anal. 5 (1995), No. 1, 76-91.

[12] Nabutovsky A. and Weinberger S., "Algorithmic unsolvability of the triviality problem for multidimensional knots", Comment. Math. Helv. 71 (1996), No. 1, 426-434.

[13] Nash J., "Real algebraic manifolds", Ann. of Math. (2) 56 (1952), No. 3, 405-421.

[14] Soare R.I., "Computability theory and differential geometry", Bull. Symbolic Logic. 10 (2004), No. 4, 457-486.

[15] Whitehead J.H.C., "On $C^{1}$-Complexes", Ann. of Math. (2) 41 (1940), No. 4, 809-824. 